\title{
Rastreamento do câncer de colo do útero em um municipio do sudoeste do Paraná
}

\author{
Cervical cancer screening in a municipality in \\ southwest Paraná
}

Bruna Carneiro Ribeiro ${ }^{1}$, Nattally Edimeire Skonieczny ${ }^{2}$, Cleunir de Fatima Candido De Bortoli $^{3}$, Gisele Iopp Massafera ${ }^{4}$

\begin{abstract}
1. Acadêmica. Graduação em Enfermagem do Centro Universitário de Pato Branco - UNIDEP. ORCID: https://orcid.org/0000-0003-1093-7377

2. Acadêmica. Graduação em Enfermagem do Centro Universitário de Pato Branco - UNIDEP. ORCID: https://orcid.org/0000-0002-1948-4717

3. Enfermeira. Mestre em Enfermagem. Professora do curso de graduação em Enfermagem Centro Universitário de Pato Branco - UNIDEP. ORCID: https://orcid.org/0000-0002-1266-5267

4. Enfermeira. Mestre em Enfermagem. Coordenadora e professora do Curso de Enfermagem do Centro Universitário de Pato Branco - PR - UNIDEP. ORCID: https://orcid.org/0000-0001-8444-7958

CONTATO: Cleunir de Fátima Candido De Bortoli | Rua Itabira, 1650- apto 702 - Centro | Pato Branco | Paraná | Telefone: (46) 98405-1666 | E-mail: cleunir_candido@hotmail.com
\end{abstract}

COMO CITAR: Ribeiro BC, Skonieczny NE, Bortoli CFC, Massafera Gl. Rastreamento do câncer de colo do útero em um município do sudoeste do Paraná. R. Saúde Públ. Paraná. 2020 Jul;3(1):41-50

COCPYGHT Esta obra é disponibilizada nos termos da Licença Creative Commons - 4 . 0
Internacional. É permitida a reprodução parcial ou total desta obra, desde que citada a fonte.

RESUMO O câncer de colo uterino é o segundo tipo de câncer mais frequente na população feminina no Brasil e apresenta um dos mais altos potenciais de prevenção e cura. O estudo teve como objetivo descrever o rastreamento do câncer de colo uterino, realizado no município de Pato Branco, no estado do Paraná, de forma descritiva, retrospectiva, no período dos anos 2015 a 2019. Realizou-se uma pesquisa de dados no Datasus, analisando a faixa etária acima de 10 anos. Nesse período, foram realizados 38.464 exames citopatológicos tendo como prevalência no grupo etário 
entre 25 e 64 anos de idade. O rastreamento foi o motivo mais indicado na realização do exame e a periodicidade de um ano entre os exames, prevaleceu em todos os anos analisados. Conclui-se que o rastreamento ocorre de forma predominante na faixa etária preconizada, ainda assim, a periodicidade encontra-se em desacordo com o recomendado.

PALAVRAS-CHAVE: Saúde da Mulher. Programas de Rastreamento. Atenção Primária à Saúde.Câncer Uterino. Papiloma vírus Humano.

\begin{abstract}
Cervical cancer is the second most common type of cancer in the female population in Brazil and presents one of the highest potentials of prevention and cure. The study aimed to describe the screening for cervical cancer, performed in the municipality of Pato Branco in the state of Paraná, in a retrospective descriptive manner from 2015 to 2019. A data survey was conducted in Datasus, analyzing the age group above 10 years. During this period, 38,464 cytopathological exams were performed, with prevalence in the age group between 25 and 64 years of age. Screening was the most mentioned reason for the examination, and the periodicity of one year between the exams prevailed in all the years analyzed. It is concluded that screening occurs predominantly in the recommended age group, yet the periodicity is in disagreement with the recommended.
\end{abstract}

Keywords: Women's Health. Mass Screening.. Primary Health Care. Uterine Neoplasms. Papillomaviridae.

\title{
INTRODUÇÃo
}

A

neoplasia de colo uterino é o segundo tipo de câncer mais frequente na população feminina no Brasil. Estimativas do Instituto Nacional de Câncer (INCA) apontam que para cada ano do triênio 2020-2022 são esperados 16.590 casos novos de câncer de colo do útero no Brasil, com um risco estimado de 15,43 casos a cada 100 mil mulheres ${ }^{12}$.

O fator de risco que prevalece para o desenvolvimento deste câncer é a presença do vírus do papiloma humano (HPV) com seus subtipos oncogênicos, sendo os de maior relevância o HPV 16 e HPV 18, sendo que o subtipo HPV 16 demonstra risco elevado. Sobretudo, outros fatores como baixa da imunidade, o tabagismo, a vida sexual precoce, a multiplicidade de parceiros sexuais, multiparidade, o uso de contraceptivos orais, a contaminação pelo Vírus da Imunodeficiência Humana (HIV) e outras infecções sexualmente transmissiveis, também levam o individuo a se tornar vulnerável. Segundo estimativas do INCA publicadas em 2019, 32\% da população de mulheres que possuem câncer de colo do útero está infectada com o tipo 16 e 18 do HPV 2.3 .

O câncer de colo uterino apresenta um dos mais altos potenciais de prevenção e cura, dentre os demais tipos de câncer, podendo ser diagnosticado na fase pré-clínica. As estratégias para a detecção precoce do câncer incluem rastreamento e diagnóstico precoce. É de grande importância a avaliação das lesões 
provocadas pelo HPV, pois quanto mais graves, maior a probabilidade de evoluírem para diagnóstico de câncer de colo uterino. 0 tumor de colo uterino se apresenta na sua fase inicial de uma forma assintomática, podendo em alguns casos manifestar pequenos sinais, fazendo com que muitas pacientes não procurem ajuda no início da doença. Por esta razão, é muito importante a vigilância continuada através de medidas de prevenção e rastreamento, sendo a segunda uma tecnologia usada pela Atenção Primária à Saúde (APS)4.

O rastreamento do câncer de colo do útero e de suas lesões precursoras deve ser realizado pelo exame citopatológico. A idade recomendada para o início da coleta deve ser aos 25 anos de idade, em mulheres que já iniciaram a atividade sexual. Quanto à periodicidade do exame, as recomendações brasileiras são: os dois primeiros exames realizados com intervalo anual e quando ambos os resultados forem negativos, os próximos devem ser realizados a cada 3 anos $^{5}$.

Neste cenário, a atenção primária possui um papel fundamental no desenvolvimento de intervenções para prevenção do câncer de colo do útero por meio de ações de educação em saúde, vacinação de grupos indicados e detecção precoce do câncer e de suas lesões precursoras por meio de seu rastreamento. 0 mesmo é uma ferramenta muito usada pela APS, em que os profissionais devem conhecer o método, população-alvo, frequência a ser realizado, sabendo ainda orientar e encaminhar para tratamento as mulheres de acordo com os resultados dos exames e garantir a continuidade do seu acompanhamento. Atingir alta cobertura da população definida como alvo é o componente mais importante no âmbito da atenção primária'.

Neste contexto, o estudo foi desenvolvido orientado pela questão de pesquisa "Como ocorre o rastreamento do câncer de colo uterino no município de Pato Branco, Paraná?", tendo como objetivo caracterizar o rastreamento do câncer de colo uterino, realizado no município de Pato Branco - Paraná.

\section{MÉTODO}

Trata-se de um estudo descritivo, retrospectivo, de abordagem quantitativa. Foi realizado no município de Pato Branco, Paraná, referente ao período de 2015 a 2019. De acordo com o Datasus (2015), a população estimada no município para o ano de 2015, era de 79.012 habitantes, sendo 40.302 mulheres. Destas, 24.916 mulheres jovens em idade fértil, cuja faixa etária correspondia a 10 e 49 anos de idade ${ }^{6}$.

Os dados foram pesquisados no Portal de Informações do Ministério da Saúde - DATASUS, através das informações do Sistema de Informação do Câncer (SISCAN), utilizando-se dados de domínio público. A população do estudo foi constituída pelo total de mulheres que realizaram o exame citopatológico de colo uterino, na rede pública de saúde do município, nos anos de 2015 a 2019. Sendo avaliada a população feminina na faixa etária acima de 10 anos, totalizando uma amostra de 38.464 exames.

Foram incluídos neste estudo, exames realizados em mulheres residentes do município de Pato Branco, Paraná. Foram excluídos nesta análise, todos as informações que constavam no banco de dados do Datasus, como branco e ignorados. Para a coleta de dados, utilizou-se de um roteiro elaborado pelas próprias pesquisadoras, contemplando as seguintes variáveis: faixa etária da realização do exame; período do exame anterior; adequabilidade da amostra; representatividade da zona de transformação.

Após a coleta, as informações foram tabuladas e apresentadas em tabelas, pela frequência absoluta e relativa dos dados. Na sequência, procedeu-se a análise descritiva destes dados.

O estudo respeitou os preceitos éticos de pesquisa envolvendo seres humanos, não utilizando nenhum tipo de identificação da população. Embasado na resolução nº 510, do Conselho Nacional de Saúde, a qual regulamenta os estudos com dados de acesso e domínio público, o estudo dispensou o registro e avaliação pelo sistema CEP/CONEP?. 


\section{RESULTADOS}

Durante o período de 2015 a 2019, foram realizados 38.464 exames citopatológicos de colo uterino em mulheres residentes de Pato Branco, Paraná. A realização da citologia prevalece no grupo etário entre 25 e 64 anos de idade, correspondendo a 75,65\% dos exames realizados. 0 grupo com idade entre 10 e 24 anos correspondeu a $18,76 \%$ dos exames e 5,59\% na idade acima de 65 anos.

Observou-se também a periodicidade do exame, na qual, em todos os anos avaliados, prevalecem os intervalos de um e dois anos entre os exames. O intervalo de três anos, recomendado nas diretrizes brasileiras, apresenta proporções inferiores, representando menos de $10 \%$ do total de exames realizados no período, como mostra a Tabela 1.

Tabela 1 - Periodicidade de realização do exame citopatológico de colo do útero de acordo com a faixa etária, no município de Pato Branco PR, 2015-2019.

\begin{tabular}{|c|c|c|c|c|}
\hline \multirow{2}{*}{ Ano } & \multirow{2}{*}{ Periodicidade } & \multicolumn{3}{|c|}{ Faixa etária } \\
\hline & & 10 a 24 anos $n(\%)$ & 25 a 64 anos n (\%) & 65 anos ou mais $n(\%)$ \\
\hline \multirow{5}{*}{2015} & Mesmo ano & $54(5,96 \%)$ & $277(5,32 \%)$ & $21(6,36 \%)$ \\
\hline & 1 ano & $530(58,50 \%)$ & $3.115(59,85 \%)$ & $174(52,73 \%)$ \\
\hline & 2 anos & 227 (25,05\%) & 1.358 (26.09\%) & 97 (29,39\%) \\
\hline & 3 anos & $75(8,28 \%)$ & $311(5,97 \%)$ & $28(8,49 \%)$ \\
\hline & 4 anos ou mais & $20(2,21 \%)$ & $144(2,77 \%)$ & $10(3,03 \%)$ \\
\hline \multirow{5}{*}{2016} & Mesmo ano & $92(8,56 \%)$ & $320(5,70 \%)$ & $21(6,02 \%)$ \\
\hline & 1 ano & $630(58,60 \%)$ & $3.483(62.03 \%)$ & $226(64,76 \%)$ \\
\hline & 2 anos & $263(24,47 \%)$ & $1.382(24,61 \%)$ & $75(21,49 \%)$ \\
\hline & 3 anos & $71(6,60 \%)$ & $310(5,52 \%)$ & $19(5,44 \%)$ \\
\hline & 4 anos ou mais & $19(1,77 \%)$ & $120(2,14 \%)$ & $8(2,29 \%)$ \\
\hline \multirow{5}{*}{2017} & Mesmo ano & $53(5,18 \%)$ & 252 (4.49\%) & $11(2,98 \%)$ \\
\hline & 1 ano & $615(60,12 \%)$ & 3.367 (59.98\%) & $216(58,54 \%)$ \\
\hline & 2 anos & $246(24,05 \%)$ & $1.470(26,18 \%)$ & 105 (28,45\%) \\
\hline & 3 anos & $85(8,31 \%)$ & $417(7,43 \%)$ & $30(8,13 \%)$ \\
\hline & 4 anos ou mais & $24(2,34 \%)$ & $108(1,92 \%)$ & $7(1,90 \%)$ \\
\hline \multirow{5}{*}{2018} & Mesmo ano & $34(3,60 \%)$ & $124(2,51 \%)$ & $1(0,26 \%)$ \\
\hline & 1 ano & $528(55,93 \%)$ & $2.810(56,88 \%)$ & 217 (55,78\%) \\
\hline & 2 anos & $265(28,07 \%)$ & $1.431(28,97 \%)$ & 122 (31,36\%) \\
\hline & 3 anos & 78 (8,27\%) & $421(8,52 \%)$ & 34 (8,74\%) \\
\hline & 4 anos ou mais & 39 (4,13\%) & $154(3,12 \%)$ & 15 (3.86\%) \\
\hline \multirow{5}{*}{2019} & Mesmo ano & 37 (3,91\%) & $140(2,58 \%)$ & $8(1,78 \%)$ \\
\hline & 1 ano & 507 (53,54\%) & $3.090(56,83 \%)$ & 252 (56,12\%) \\
\hline & 2 anos & 293 (30,94\%) & $1.501(27,61 \%)$ & $118(26,28 \%)$ \\
\hline & 3 anos & 79 (8,34\%) & 470 (8,64\%) & 43 (9,58\%) \\
\hline & 4 anos ou mais & 31 (3,27\%) & $236(4,34 \%)$ & $28(6,24 \%)$ \\
\hline
\end{tabular}


Analisando os motivos da realização do exame, mais de $98 \%$ foram de rastreamento. Sendo que a repetição após o exame alterado e seguimento, corresponde a menos de $2 \%$ dos exames realizados, como apresenta a Tabela 2.

Tabela 2 - Distribuição de exames citopatológicos de colo do útero realizados por ano, segundo faixa etária e motivo de indicação, no município de Pato Branco - PR, 2015-2019.

\begin{tabular}{|c|c|c|c|c|c|c|c|c|c|}
\hline \multicolumn{10}{|c|}{ MOTIVO DO EXAME DE ACORDO COM A FAIXA ETÁRIA E PERIODO } \\
\hline \multirow{3}{*}{ Ano } & \multicolumn{3}{|c|}{ Rastreamento } & \multicolumn{3}{|c|}{ Repetição } & \multicolumn{3}{|c|}{ Seguimento } \\
\hline & 10 a 24 & 25 a 64 & $\geq 65$ & 10 a 24 & 25 a 64 & $\geq 65$ & 10 a 24 & 25 a 64 & $\geq 65$ \\
\hline & \multicolumn{3}{|c|}{$\begin{array}{c}n \\
(\%)\end{array}$} & \multicolumn{3}{|c|}{$\begin{array}{c}n \\
(\%)\end{array}$} & \multicolumn{3}{|c|}{$\begin{array}{c}n \\
(\%)\end{array}$} \\
\hline 2016 & $\begin{array}{c}1.590 \\
(99,25)\end{array}$ & $\begin{array}{c}6.076 \\
(98,78)\end{array}$ & $\begin{array}{c}425 \\
(99,76)\end{array}$ & $\begin{array}{c}10 \\
(0,62)\end{array}$ & $\begin{array}{c}52 \\
(0,84)\end{array}$ & 0 & $\begin{array}{c}2 \\
(0,13)\end{array}$ & $\begin{array}{c}23 \\
(0,38)\end{array}$ & $\begin{array}{c}1 \\
(0,24)\end{array}$ \\
\hline 2018 & $\begin{array}{c}1.316 \\
(99,10)\end{array}$ & $\begin{array}{c}5.238 \\
(99,47)\end{array}$ & $\begin{array}{c}424 \\
(99,77)\end{array}$ & $\begin{array}{c}5 \\
(0,38)\end{array}$ & $\begin{array}{c}13 \\
(0,25)\end{array}$ & 0 & $\begin{array}{c}7 \\
(0,52)\end{array}$ & $\begin{array}{c}15 \\
(0,28)\end{array}$ & $\begin{array}{c}1 \\
(0,23)\end{array}$ \\
\hline 2019 & $\begin{array}{c}1.355 \\
(99,86)\end{array}$ & $\begin{array}{c}5.801 \\
(99,42)\end{array}$ & $\begin{array}{c}485 \\
(100)\end{array}$ & $\begin{array}{c}1 \\
(0,07)\end{array}$ & $\begin{array}{c}18 \\
(0,34)\end{array}$ & 0 & $\begin{array}{c}1 \\
(0,07)\end{array}$ & $\begin{array}{c}16 \\
(0,24)\end{array}$ & 0 \\
\hline
\end{tabular}

Fonte: Elaborada pelos autores (2020)

Referente à adequabilidade da amostra, o período pesquisado revelou que apenas duas amostras no ano de 2019 foram rejeitadas, ambas na faixa etária de 25 a 64 anos. Os números de amostras insatisfatórias são relativamente pequenos, prevalecendo a maioria das amostras classificadas como satisfatórias, correspondendo a mais de 99\% do total de exames realizados, como apresentados na Tabela 3.

Analisando o percentual de amostras que apresentavam epitélio representativo da zona de transformação, os achados do presente estudo mantiveram-se abaixo do esperado em todos os períodos analisados. Mantiveram um crescimento ascendente em todas as faixas etárias entre 2015 e 2018 e em 2019 voltou a apresentar-se decrescente. A menor representatividade do epitélio da zona de transformação esteve no grupo acima de 65 anos de idade, como revela a Tabela 4. 
Tabela 3 - Distribuição dos exames realizados, quanto à adequabilidade da amostra, segundo a faixa etária e período analisado, no município de Pato Branco - PR, 2015-2019.

\begin{tabular}{|c|c|c|c|c|c|c|}
\hline \multicolumn{7}{|c|}{ ADEQUABILIDADE DA AMOSTRA } \\
\hline \multirow{3}{*}{ Ano } & \multicolumn{3}{|c|}{ Satisfatória } & \multicolumn{3}{|c|}{ Insatisfatória } \\
\hline & 10 a 24 & 25 а 64 & $\geq 65$ & 10 a 24 & 25 а 64 & $\geq 65$ \\
\hline & $\begin{array}{c}n \\
(\%)\end{array}$ & $\begin{array}{c}n \\
(\%)\end{array}$ & $\begin{array}{c}n \\
(\%)\end{array}$ & $\begin{array}{c}\mathrm{n} \\
(\%)\end{array}$ & $\begin{array}{c}n \\
(\%)\end{array}$ & $\begin{array}{c}n \\
(\%)\end{array}$ \\
\hline 2015 & $\begin{array}{c}1.384 \\
(99,92)\end{array}$ & $\begin{array}{c}5.783 \\
(99,91)\end{array}$ & $\begin{array}{c}395 \\
(100)\end{array}$ & $\begin{array}{c}1 \\
(0,08)\end{array}$ & $\begin{array}{c}5 \\
(0,08)\end{array}$ & 0 \\
\hline 2016 & $\begin{array}{c}1.601 \\
(99,92)\end{array}$ & $\begin{array}{c}6.149 \\
(99,96)\end{array}$ & $\begin{array}{c}424 \\
(99,53)\end{array}$ & $\begin{array}{c}1 \\
(0,08)\end{array}$ & $\begin{array}{c}2 \\
(0,04)\end{array}$ & $\begin{array}{c}2 \\
(0,47)\end{array}$ \\
\hline 2017 & $\begin{array}{c}1.544 \\
(99,92)\end{array}$ & $\begin{array}{c}6.048 \\
(99,85)\end{array}$ & $\begin{array}{c}419 \\
(100)\end{array}$ & $\begin{array}{c}1 \\
(0,08)\end{array}$ & $\begin{array}{c}9 \\
(0,15)\end{array}$ & 0 \\
\hline 2018 & $\begin{array}{c}1.327 \\
(99,92)\end{array}$ & $\begin{array}{c}5.260 \\
(99,88)\end{array}$ & $\begin{array}{c}425 \\
(100)\end{array}$ & $\begin{array}{c}1 \\
(0,08)\end{array}$ & $\begin{array}{c}6 \\
(0,12)\end{array}$ & 0 \\
\hline 2019 & $\begin{array}{l}1.357 \\
(100)\end{array}$ & $\begin{array}{l}5.820 \\
(99,74)\end{array}$ & $\begin{array}{c}483 \\
(99,58)\end{array}$ & 0 & $\begin{array}{c}11 \\
(0,18)\end{array}$ & $\begin{array}{c}2 \\
(0,42)\end{array}$ \\
\hline
\end{tabular}

Fonte: Elaborada pelos autores (2020).

Tabela 4 - Representatividade da zona de transformação do exame citopatológico de colo do útero segundo faixa etária e período, no município de Pato Branco - PR, 2015-2019.

\begin{tabular}{|c|c|c|c|c|c|c|c|c|c|}
\hline \multirow[b]{3}{*}{ Ano } & \multicolumn{9}{|c|}{ REPRESENTATIVIDADE DA ZONA DE TRANSFORMAÇÃO DO EXAME CITOPATOLÓGICO } \\
\hline & \multicolumn{3}{|c|}{10 a 24} & \multicolumn{3}{|c|}{25 a 64} & \multicolumn{3}{|c|}{$\geq 65$} \\
\hline & $\mathrm{n}$ & $\%$ & $\begin{array}{c}\text { Total de } \\
\text { amostras }\end{array}$ & $\mathrm{n}$ & $\%$ & $\begin{array}{c}\text { Total de } \\
\text { amostras }\end{array}$ & $\mathrm{n}$ & $\%$ & $\begin{array}{c}\text { Total de } \\
\text { amostras }\end{array}$ \\
\hline 2015 & 698 & 50,39 & 1.385 & 2.373 & 40,99 & 5.788 & 46 & 11,64 & 395 \\
\hline 2016 & 972 & 60,67 & 1.602 & 3.191 & 51,87 & 6.151 & 73 & 17,13 & 426 \\
\hline 2017 & 1.160 & 75,08 & 1.545 & 4.180 & 69.01 & 6.057 & 191 & 45,58 & 419 \\
\hline 2018 & 1.148 & 86,44 & 1.328 & 4.140 & 78,61 & 5.266 & 201 & 47,29 & 425 \\
\hline 2019 & 874 & 64,40 & 1.357 & 3.253 & 55,74 & 5.835 & 150 & 30,92 & 485 \\
\hline
\end{tabular}

Legenda: $n$ - frequência absoluta das amostras com epitélio representativo da zona de transformação; \% - frequência relativa das amostras com epitélio representativo da zona de transformação. Fonte: Elaborada pelos autores (2020).

\section{DISCUSSÃo}

Os resultados do estudo apontam um percentual significativo de exames realizados fora do grupo etário recomendado, considerado como população alvo do rastreamento. Revelou ainda, que os intervalos entre os exames realizados, em sua maioria, não seguem as recomendações das diretrizes nacionais. Considerando 
que o motivo da indicação do exame em mais de $98 \%$ foi o rastreamento, o intervalo esperado, neste caso, é de três anos entre os exames 5 .

No Brasil, o rastreamento do câncer de colo uterino ocorre predominantemente de forma oportunista, no qual a mulher realiza o exame ao procurar os serviços de saúde por outras razões. Com isso, uma parcela dos exames realizados, $20 \%$ a $25 \%$, ocorrem em uma faixa etária fora do recomendado e em torno da metade deles com intervalo de um ano ou menos, quando o preconizado são três anos. Isso, por sua vez. resulta em um contingente de mulheres super-rastreadas e outro, sem a realização de qualquer exame de rastreamento ${ }^{5}$.

O desenvolvimento do câncer de colo uterino em mulheres até 24 anos é muito baixo e, por isso, o rastreamento nesta faixa etária não é considerado eficaz para a detecção. Além disso, o tratamento de lesões precursoras em adolescentes e mulheres jovens eleva o número de colposcopias, aumentando consequentemente a morbidade obstétrica e neonatal em futuras gestações, considerando que a maioria das mulheres nesta idade não possui sua prole definida. Ressalta-se, ainda, o impacto psíquico que o diagnóstico de uma doença sexualmente transmissível e precursora do câncer acarretará em adolescentes e mulheres adultas jovens sobre a sua sexualidade e autoimagem. Para tanto, esses fatores justificam a redução de intervenções no colo do útero em mulheres jovens ${ }^{5-8}$.

Estudo de abrangência nacional pesquisando a realização do exame Papanicolau nos últimos três anos, revelou uma cobertura do exame abaixo de $80 \%$ entre mulheres com 25 e 64 anos de idade. Identificou, ainda, importantes diferenças regionais na cobertura de realização do exame ${ }^{9}$.

Por outro lado, a disponibilidade do exame nas Unidades Básicas de Saúde, a reorganização das redes de prevenção, diagnóstico e tratamento do câncer de colo uterino são primordiais para a cobertura e o alcance da meta almejada. Ainda, autores reforçam a significância de evoluir para um modelo de rastreamento organizado, substituindo o atualmente predominante, nominado como oportunístico.

Um modelo de rastreamento organizado contempla também seguir a periodicidade do exame preconizado. No presente estudo, evidenciou-se índices elevados de intervalos de um e dois anos entre os exames. Entretanto, para manter o intervalo trienal entre os exames, há que se garantir a qualidade das amostras coletadas. Quanto à adequabilidade das amostras no período pesquisado, acima de $99 \%$ foram consideradas satisfatórias, menos de 1\% foram insatisfatórias e apenas duas amostras foram rejeitas no período pesquisado.

Uma amostra é considerada satisfatória quando apresenta células em quantidade representativa, bem distribuídas, fixadas e coradas, permitindo que em sua observação seja possível emitir uma conclusão diagnóstica. Pode ser considerada insatisfatória a amostra que em sua leitura e avaliação seja prejudicada por algumas situações, como material acelular ou hipocelular ou prejudicada pela presença de sangue, piócitos, artefatos de dessecamento, contaminantes externos ou acentuada superposição celular. Nestes casos, o exame deverá ser repetido em seis a doze semanas, com a correção prévia do problema, quando possível'.

Ao avaliar a representatividade do epitélio da amostra, importante indicador da qualidade da coleta do exame, evidenciou-se um percentual muito baixo, considerando o ano de 2015 com os piores resultados, atingindo apenas 11,64\% dos exames na faixa etária acima de 65 anos, 40,99\% das mulheres entre 25 a 64 anos e 50,39\% no grupo até 24 anos. Nos demais períodos analisados, observou-se um aumento significativo da representatividade da Zona de Transformação, especialmente entre as mulheres mais jovens.

Esses achados corroboram com os resultados de estudo realizado analisando as amostras de mulheres com 40 anos ou mais, nas quais a presença de células representativas da junção escamocolunar (JEC) 
diminuiu de forma progressiva com o passar da idade. No grupo entre 40 e 50 anos, $44,3 \%$ dos exames não apresentavam JEC nos esfregaços; entre 51 e 60 anos, 48\% não apresentavam; entre 61 a 70 anos, 56,6\% não apresentavam, e na faixa etária acima de 70 anos, 69,5\% não apresentavam a JEC ${ }^{10}$.

A presença dos epitélios representados na amostra, considerada como informação obrigatória nos laudos citopatológicos, é de responsabilidade do profissional que realizou a coleta do material. A identificação de células representativas da JEC revela que, durante a coleta, buscou-se obter elementos celulares representativos do local onde praticamente a totalidade dos casos de canceres de colo uterino se desenvolvem?

Estudos avaliando a eficácia de diferentes dispositivos de coleta, revelou que o uso da espátula de Ayre e da escova endocervical elevam em até três vezes a possibilidade de obter células endocervicais. A exclusividade da presença de células escamosas deve ser avaliada pelo médico assistente da mulher. Cabe ressaltar que é fundamental que os profissionais de saúde estejam atentos para a representatividade da JEC nas amostras, pois a sua ausência pode deixar de proporcionar todos os benefícios da prevenção do câncer de colo uterino à mulher'

Neste contexto, o uso correto da espátula de Ayre e da escovinha são fundamentais para obter uma amostragem de qualidade, assim como a fixação correta e imediata do material coletado. Para tanto, autores reforçam que apesar de todos os benefícios do exame preventivo, possiveis falhas na coleta do material, no preparo, na conservação e interpretação citopatológica, podem comprometer a eficácia do rastreamento do câncer de colo do útero"1. Autores apontam a necessidade de maior qualificação profissional, tanto em nível de atenção primária à saúde, responsável pelo rastreamento, incluindo a coleta do exame citopatológico de colo uterino, assim como dos profissionais atuantes nos laboratórios, a quem cabe a responsabilidade de leitura e análise dos esfregaços ${ }^{12}$.

Contudo, estudo de avaliação de esfregaços cervicovaginais revelou que as mulheres com 40 anos ou mais apresentam uma incidência menor de JEC em amostras avaliadas. Enquanto a maioria das mulheres com idade inferior a 40 anos, sem material JEC, 74,5\% apresentavam inflamação exuberante no esfregaço. Entretanto, a presença de um processo inflamatório moderado a intenso é apontado como um fator limitante para uma coleta de amostra de qualidade em usuárias com idade inferior a 40 anos. Desta forma, a mulheres apresentando quadro inflamatório acentuado, identificado durante o exame ginecológico, propõese primeiramente o tratamento adequado, para posterior coleta de exame citológico ${ }^{12}$

Neste contexto, destaca-se também a realização do exame citopatológico de colo uterino em mulheres histerectomizadas, cujo colo uterino está ausente. As recomendações brasileiras orientam que, mulheres que foram submetidas a histerectomia total por lesões benignas, sem histórico anterior de lesões cervicais de alto grau, cujos exames anteriores normais, podem ser excluídas do rastreamento'.

\section{CONCLUSÃO}

A análise dos dados conclui que o rastreamento no cenário pesquisado é realizado como o predominante no território brasileiro, de forma oportunística. A realização do exame ocorre de forma predominante na faixa etária preconizada nas diretrizes brasileiras. No entanto, a periodicidade do exame encontra-se aquém do recomendado, prevalecendo o intervalo de um e dois anos, em todos os períodos avaliados.

Para um rastreamento organizado, há que se dispor de muito além da realização do exame na faixa 
etária recomendada e no intervalo de tempo preconizado. Faz-se necessária uma coleta do material de forma adequada, assim como do controle de qualidade dos serviços responsáveis pela leitura e análise das amostras.

Evidenciou-se, através deste estudo, quase a totalidade das amostras classificadas como satisfatórias para a análise, o que demonstra um esfregaço de boa qualidade, bem fixado, identificado corretamente e livre de contaminantes externos.

Vale destacar uma variável importante ao que se refere à qualidade da coleta da amostra, que é a representatividade do epitélio da zona de transformação. No presente estudo, mostrou-se uma baixa frequência, fato que pode estar relacionado a diversos fatores, como a qualidade da coleta, a realização do exame em mulheres histerectomizadas e a realização do exame em mulheres na pós-menopausa.

Para tanto, propõe-se que sejam contempladom nas ações de educação permanente, momentos de qualificação dos profissionais que realizam a coleta do exame e a análise do resultado, para que estejam atentos a essa informação, oportunizando à mulher um rastreamento adequado.

Espera-se que as contribuições deste estudo possam refletir nas ações desenvolvidas pelas equipes de saúde, em especial nas práticas do enfermeiro, no rastreamento do câncer de colo uterino e na formação de novos profissionais. Considera-se como limitação deste estudo, o uso de dados secundários oriundos de sistemas de informação, os quais estão sujeitos à falhas na sua alimentação.

\section{REFERÊNCIAS}

1. INCA. Instituto Nacional de Câncer Jose Alencar Gomes da Silva. Diretrizes brasileiras para o rastreamento do câncer do colo do útero. 2. ed. rev. atual. Rio de Janeiro. 2016.

2. INCA. Instituto Nacional de Câncer José Alencar Gomes da Silva. Estimativa: incidência de câncer no Brasil. Rio de Janeiro. 2019. Disponivel: https://www.inca.gov.br/sites/ufu.sti.inca.local/files//media/document//estimativa-2020-incidencia-de-cancer-no-brasil.pdf

3. FEBRASGO. Federação Brasileira das Associações de Ginecologia e Obstetrícia. Rastreio, diagnóstico e tratamento do câncer de colo de útero. São Paulo, 2017.

4. INCA. Instituto Nacional de Câncer José Alencar Gomes da Silva. Exposição a Mulher e o Câncer de Colo do Útero, 2018.

5. INCA. Instituto Nacional de Câncer José Alencar Gomes da Silva. Parâmetros técnicos para o rastreamento do câncer de colo do útero. Rio de Janeiro: INCA. 2019.

6. DATASUS - Departamento de Informática do Sistema Único de Saúde. População residente - estudo de estimativas populacionais por município, idade e sexo 2000-2015. [Citado em 2020 Mai 31]. Disponível em: https://tabnet.datasus.gov.br/cgi/tabcgi.exe?novapop/cnv/ popbr.def

7. BRASIL. Resolução n 510, de 07 de abril de 2016, do Conselho Nacional de Saúde. Dispõe sobre as normas aplicáveis a pesquisas em Ciências Humanas e Sociais. Brasilia: Conselho Nacional de Saúde. 2016.

8. Conde CR, Lemos TMR, Ferreira MLSM. Características sociodemográficas, individuales y de programación de las mujeres con cáncer cervical. Enferm Glob. [Internet] 2018. [Citado em 2020 mar 31];17(49):348-80. Disponível em: http://dx.doi.org/10.6018/eglobal.17.1.301041.

9. Oliveira MM, Andrade SSCA, Oliveira PPV, Silva GA, SILVA MMA, Malta DC. Cobertura de exame Papanicolau em mulheres de 25 a 64 anos, segundo a Pesquisa Nacional de Saúde e o Sistema de Vigilância de Fatores de Risco e Proteção para Doenças Crônicas por Inquérito Telefônico, 2013. Rev Bras Epidemiol. [Internet]. 2018 [Citado em 2020 mar 12];21:e180014. Disponivel em: http://www.scielo.br/scielo. php?script=sci_arttext\&pid=S1415-790X2018000100413\&lng=en.

10. Nai GA, Souza KKGS, Rodrigues ER, Barbosa RL. Presença de células da junção escamo-colunar em esfregaços cérvico-vaginais de mulheres acima de 40 anos. Rev Bras Ginecol Obstet. [Internet]. 2011 Mar [Citado em 2020 Mar 31];33(3):128-32. Disponivel em: <http://www. scielo.br/scielo.php?script=sci_arttext\&pid=S0100-72032011000300005\#end>

11. Machado EP, Wosniack C, Reche PM, Costa BR, Santos KMB, Perek KV, et al. Projeto Extensionista: uma Abordagem Interdisciplinar junto à Enfermagem na Prevenção do Câncer do Colo Uterino. Rev Extensão em Foco. [Internet]. 2018 [Citado em 2020 Mar 31];16:12-25. Disponível em: <https://revistas.ufpr.br/extensao/article/view/56351> 
12. Damacena AM, Luz LL, Mattos IE. Rastreamento do câncer do colo do útero em Teresina, Piauí: estudo avaliativo dos dados do Sistema de Informação do Câncer do Colo do Útero, 2006-2013. Epidemiol Serv Saúde. [Internet] 2017 [Citado em 2020 mar 31];26(1):71-80. Disponivel em : <http://www.scielo.br/scielo.php?pid=S2237-96222017000100071\&script=sci_abstract\&tlng=pt>.

RECEBIDO: 31/03/2020

ACEITO: $18 / 06 / 2020$ 